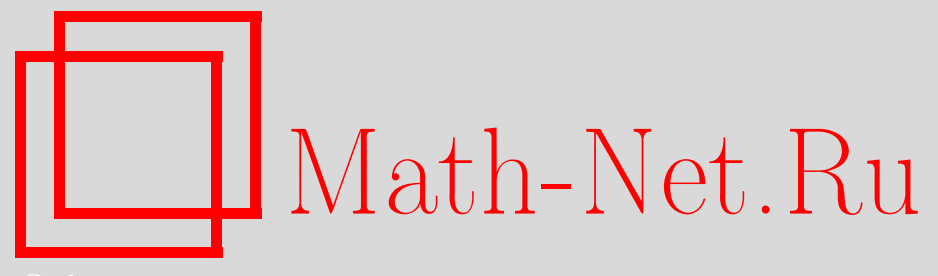

В. А. Колмыков, О характеристических многочленах периодических графов, Дискрет. матем., 2004, том 16, выпуск $3,153-159$

DOI: https://doi.org/10.4213/dm169

Использование Общероссийского математического портала Math-Net.Ru подразумевает, что вы прочитали и согласны с пользовательским соглашением http://www.mathnet.ru/rus/agreement

Параметры загрузки:

IP : 54.174 .149 .18

26 апреля 2023 г., 05:39:23

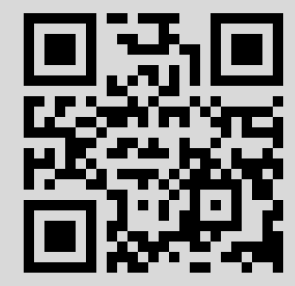


УдК 519.17

\title{
О характеристических многочленах периодических графов
}

\author{
() 2004 г. В. А. Колмыков
}

\begin{abstract}
Рассматриваются два типа периодических графов, цепное и циклическое продолжения произвольного графа. Находятся формулы, выражающие характеристические многочлены таких конструкций, через многочлены основного графа, его подграфов и многочлены цепи и цикла. Проиллюстрированы некоторые применения этих результатов.
\end{abstract}

\section{1. Характеристический многочлен графа}

В статье рассматриваются конечные графы без петель и кратных ребер. Несмотря на известную узость такого класса графов, он, однако, оказывается вполне достаточным для большинства вопросов спектральной теории графов и ее приложений в химии.

Характеристический многочлен матрицы смежности графа $G$ не зависит от нумерации вершин, он называется характеристическим многочленом графа и обозначается $G(\lambda)$, характеристический многочлен пустого графа по определению тождественно равен единище. Подробнее об этом см. [1].

Двухкорневым графом с упорядоченными корнями называется граф, в котором отмечены две вершины, эти вершины называются корнями, причем один из корней считается первым, другой вторым. Пусть $G$ - двухкорневой граф с упорядоченными корнями. Первый корень $G$ обычно обозначают $\alpha(G)$ или просто $\alpha$, если ясно, о каком графе идет речь. Второй корень графа $G$ обозначают $\beta(G)$ или просто $\beta$.

Пусть $U$ - некоторое подмножество множества вершин графа $G$, тогда $G_{U}$ означает граф, получающийся из $G$ удалением всех вершин множества $U$ (например, $G_{\alpha}$ означает граф, в котором удален первый корень). Если $F$ - подграф графа $G$ и $V(F)-$ множество всех вершин подграфа $F$, то $G_{V(F)}$ коротко обозначается $G_{F}$.

Для двухкорневого графа $G$ с упорядоченными корнями положим

$$
\begin{aligned}
G_{\alpha * \beta}(\lambda) & =G_{\alpha}(\lambda)+G_{\beta}(\lambda)-\lambda G_{\alpha \beta}(\lambda), \\
G_{[\alpha \beta]}(\lambda) & =\sum_{\alpha \cdot P \cdot \beta} G_{P}(\lambda),
\end{aligned}
$$

где суммирование ведется по всем вершинно-простым путям $P$ с концами $\alpha$ и $\beta$. В [2] доказано, что

$$
G_{[\alpha \beta]}(\lambda)=\sqrt{G_{\alpha}(\lambda) G_{\beta}(\lambda)-G(\lambda) G_{\alpha \beta}(\lambda)} .
$$

Здесь символ корня означает арифметический квадратный корень в кольце многочленов, то есть $\sqrt{f}=g \Longleftrightarrow f=g^{2}$ и старший коэффищиент $g$ неотрищателен. 


\section{2. Периодические графы}

На множестве двухкорневых графов с упорядоченными корнями определена ассоциативная операция склейки о следующим образом: $G \circ F$ получается из $G$ и $F$ отождествлением $\beta(G)$ и $\alpha(F)$, причем по определению

$$
\alpha(G \circ F)=\alpha(G), \quad \beta(G \circ F)=\beta(F) .
$$

Если $H-$ двухкорневой граф с упорядоченными корнями, то граф $H \circ H \circ \ldots$. $H$ (c $n-1$ операцией склейки) называется периодическим цепным графом и обозначается $P_{n}[H]$. Это двухкорневой граф с упорядоченными корнями. Если $n \geqslant 3$, то граф, получающийся из $P_{n}[H]$ с помощью отождествления его корней, называется периодическим циклическим графом и обозначается $C_{n}[H]$.

Ниже мы используем стандартные обозначения $P_{k}$ и $C_{k}$ соответственно для $k$-вершинной цепи и $k$-вершинного цикла. Кроме того, удобно положить $P_{0}(\lambda) \equiv 1$ и $P_{-1}(\lambda) \equiv 0$.

Теорема 1. Пусть $H$ - двухкорневой граф с упорядоченными корнями.

Для тех $\lambda$, для которых $H_{[\alpha \beta]}(\lambda) \neq 0$, справедливы равенства

$$
\begin{aligned}
& P_{n}[H](\lambda)=\left(H_{[\alpha \beta]}(\lambda)\right)^{n-1} P_{n-1}\left(\frac{H_{\alpha * \beta}(\lambda)}{H_{[\alpha \beta]}(\lambda)}\right) H(\lambda)-\lambda\left(H_{[\alpha \beta]}(\lambda)\right)^{n} P_{n-2}\left(\frac{H_{\alpha * \beta}(\lambda)}{H_{[\alpha \beta]}(\lambda)}\right), \\
& C_{n}[H](\lambda)=\left(H_{[\alpha \beta]}(\lambda)\right)^{n} C_{n}\left(\frac{H_{\alpha * \beta}(\lambda)}{H_{[\alpha \beta]}(\lambda)}\right) .
\end{aligned}
$$

Для тех $\lambda$, для которых $H_{[\alpha \beta]}(\lambda)=0$, справедливы равенства

$$
\begin{aligned}
& P_{n}[H](\lambda)=\left(H_{\alpha * \beta}(\lambda)\right)^{n-1} H(\lambda), \\
& C_{n}[H](\lambda)=\left(H_{\alpha * \beta}(\lambda)\right)^{n} .
\end{aligned}
$$

Отметим, что утверждение о $P_{n}[H]$ доказано совместно с В. Ф. Субботиным.

Доказательство. В спектральной теории графов хорошо известна (независимо доказанная многими авторами) теорема о разрезании по разделяющей вершине. Сформулируем еe.

Пусть в графе $G$ есть разделяющая вершина. Тогда $G$ можно представить в виде склейки двух графов $A$ и $B$ посредством отождествления некоторой вершины $u$ графа $A$ и некоторой вершины $v$ графа $B$. Теорема о разрезания утверждает, что

$$
G(\lambda)=A_{u}(\lambda) B(\lambda)+A(\lambda) B_{v}(\lambda)-\lambda A_{u}(\lambda) B_{v}(\lambda)
$$

Теорему о разрезании нам удобно использовать в матричном виде, причем в ситуации, когда граф описан как склейка двух двухкорневых графов $E$ и $F$ :

$$
E \circ F(\lambda)=\left(\begin{array}{ll}
E(\lambda) & E_{\beta}(\lambda)
\end{array}\right)\left(\begin{array}{cc}
0 & 1 \\
1 & -\lambda
\end{array}\right)\left(\begin{array}{c}
F(\lambda) \\
F_{\alpha}(\lambda)
\end{array}\right) .
$$

Положим

$$
M_{n}=\left(\begin{array}{cc}
P_{n}[H](\lambda) & P_{n}[H]_{\beta}(\lambda) \\
P_{n}[H]_{\alpha}(\lambda) & P_{n}[H]_{\alpha \beta}(\lambda)
\end{array}\right), \quad \Lambda=\left(\begin{array}{cc}
0 & 1 \\
1 & -\lambda
\end{array}\right)
$$


Применяя теорему о разрезании, получим, что $M_{m+k}=M_{m} \Lambda M_{k}$. Отсюда следует, что

$$
M_{n}=M_{1} \Lambda M_{1} \Lambda \ldots \Lambda M_{1}=\left(M_{1} \Lambda\right)^{n} \Lambda^{-1}
$$

Степень квадратной матрицы второго порядка может быть выражена через саму эту матрицу и многочлены Чебышева второго рода

$$
U_{0}(x)=1, \quad U_{1}(x)=2 x, \quad U_{n+1}(x)=2 x U_{n}(x)-U_{n-1}(x), \quad n \in \mathbf{N} .
$$

Если позволить индексу $\boldsymbol{n}$ принимать произвольные целые значения, то при таком доопределении $U_{-1}(x)=0$.

Лемма 1. Для любой комплексной $2 \times 2$ матричы $M$ и натурального $n$

$$
M^{n}=(\sqrt{\operatorname{det} M})^{n-1} U_{n-1}\left(\frac{\operatorname{tr} M}{2 \sqrt{\operatorname{det} M}}\right) M-(\sqrt{\operatorname{det} M})^{n} U_{n-2}\left(\frac{\operatorname{tr} M}{2 \sqrt{\operatorname{det} M}}\right) I,
$$

если $\operatorname{det} M \neq 0$;

$$
M^{n}=(\operatorname{tr} M)^{n-1} M,
$$

eсли $\operatorname{det} M=0$.

Здесь символ корня обозначает арифметический корень в С, т.е. $\sqrt{z}=w \Longleftrightarrow z=w^{2}$ $u$ arg $w \in(-\pi / 2, \pi / 2]$.

Доказательство. Для доказательства используется индукция по $n$. Однако индукция не объясняет происхождения формулы. Приведем наброски двух естественных доказательств. Первое использует приведение $M$ к жордановой форме $M=L J L^{-1}$. Тогда $M^{n}=L J^{n} L^{-1}$. Затем можно воспользоваться тем, что

$$
\left(\left(x+\sqrt{x^{2}-1}\right)^{k}-\left(x-\sqrt{x^{2}-1}\right)^{k}\right) /\left(2 \sqrt{x^{2}-1}\right)=U_{k-1} .
$$

Такое доказательство приводит к многочленам Чебышева. Неудобно оно тем, что требует рассмотрения случаев диагонализируемости и недиагонализируемости, либо в случае недиагонализируемости приходится воспользоваться предельным переходом по множеству диагонализируемых матриц. Второе доказательство использует Фробениусову рекурсию $M^{k}\left(M^{2}-M \operatorname{tr} M+I \operatorname{det} M\right)=0$. Затем рекурсия $a_{k+2}-t a_{k+1}+d a_{k}=0$ приводится к чебышевской.

Перейдем к доказательству индукщией по $n$. Положим

$$
\begin{aligned}
t & =\operatorname{tr}\left(M_{1} \Lambda\right)=H_{\alpha}(\lambda)+H_{\beta}(\lambda)-\lambda H_{\alpha \beta}(\lambda), \\
d & =\operatorname{det}\left(M_{1} \Lambda\right)=H_{\alpha}(\lambda) H_{\beta}(\lambda)-H(\lambda) H_{\alpha \beta}(\lambda), \\
s & =H_{[\alpha \beta]}(\lambda) .
\end{aligned}
$$

Для тех $\lambda$, для которых $d \neq 0$, справедливы равенства

$$
M_{n}=(\sqrt{d})^{n-1} U_{n-1}\left(\frac{t}{2 \sqrt{d}}\right) M_{1}-(\sqrt{d})^{n} U_{n-2}\left(\frac{t}{2 \sqrt{d}}\right) \Lambda^{-1},
$$

а для тех $\lambda$, для которых $d=0$,

$$
M_{n}=t^{n-1} M_{1}
$$


Рассмотрим случай, когда $\lambda$ вещественное. Тогда $\sqrt{d}=|s|$, так как $d=s^{2}$. Функция $U_{k}$ четна или нечетна вместе с $k$, поэтому $|b|^{k} U_{k}(a /|b|)=b^{k} U_{k}(a / b)$. Кроме того, $U_{k}(x / 2) \equiv P_{k}(x)$ (см. [1], §2.6, п. 7).

Таким образом, для тех вещественных $\lambda$, для которых $s \neq 0$,

$$
M_{n}=s^{n-1} P_{n-1}(t / s) M_{1}-s^{n} P_{n-2}(t / s) \Lambda^{-1},
$$

а для тех вещественных $\lambda$, для которых $s=0$,

$$
M_{n}=t^{n-1} M_{1} \text {. }
$$

Эти соотношения верны и в случае произвольного комплексного $\lambda$, так как они состоят из дробно-рациональных выражений.

Матричные равенства (1) и (2) распишем поэлементно. Для тех $\lambda$, для которых $s \neq 0$,

$$
\begin{aligned}
P_{n}[H](\lambda) & =s^{n-1} P_{n-1}(t / s) H(\lambda)-s^{n} P_{n-2}(t / s) \lambda, \\
P_{n}[H]_{\beta}(\lambda) & =s^{n-1} P_{n-1}(t / s) H_{\beta}(\lambda)-s^{n} P_{n-2}(t / s), \\
P_{n}[H]_{\alpha}(\lambda) & =s^{n-1} P_{n-1}(t / s) H_{\alpha}(\lambda)-s^{n} P_{n-2}(t / s), \\
P_{n}[H]_{\alpha \beta}(\lambda) & =s^{n-1} P_{n-1}(t / s) H_{\alpha \beta}(\lambda) .
\end{aligned}
$$

Для тех $\lambda$, для которых $s=0$,

$$
\begin{aligned}
P_{n}[H](\lambda) & =t^{n-1} H(\lambda), \\
P_{n}[H]_{\beta}(\lambda) & =t^{n-1} H_{\beta}(\lambda), \\
P_{n}[H]_{\alpha}(\lambda) & =t^{n-1} H_{\alpha}(\lambda), \\
P_{n}[H]_{\alpha \beta}(\lambda) & =t^{n-1} H_{\alpha \beta}(\lambda)
\end{aligned}
$$

Равенства (3) и (7) - это те утверждения о $P_{n}[H]$, которые требовалось доказать.

Из (4), (5), (6) и (8), (9), (10) получаем, что для тех $\lambda$, для которых $s \neq 0$, выполнено равенство

$$
P_{n}[H]_{\alpha * \beta}(\lambda)=\left(P_{n-1}(t / s) t / s-2 P_{n-2}(t / s)\right) s^{n},
$$

а для тех $\lambda$, для которых $s=0$,

$$
P_{n}[H]_{\alpha * \beta}(\lambda)=t^{n} .
$$

Теперь рассмотрим граф $C_{n}[H]$. Напомним формулу Швенка (см. [1], §2.7, п. 9)

$$
G(\lambda)=\lambda G_{v}(\lambda)-\sum_{u \sim v} G_{u v}(\lambda)-2 \sum_{\mathscr{C} \ni v} G_{\mathscr{C}}(\lambda) .
$$

Первое суммирование ведется по всем вершинам $u$, смежным с $v$, второе - по всем простым (вершинно-простым) циклам $\mathscr{C}$, содержащим вершину $v$.

Пример 1. Справедливы равенства

$$
C_{n}(\lambda)=\lambda P_{n-1}(\lambda)-2 P_{n-2}(\lambda)-2 .
$$

Раскроем $C_{n}[H](\lambda)$ по формуле Швенка, применив ее к вершине (обозначим ее $v$ ), полученной отождествлением корней графа $P_{n}[H]$. Тогда

$$
C_{n}[H](\lambda)=\lambda C_{n}[H]_{v}(\lambda)-\sum_{u \sim v} C_{n}[H]_{u v}(\lambda)-2 \sum_{\mathscr{C} \ni v} C_{n}[H]_{\mathscr{C}}(\lambda) .
$$


Множество простых циклов графа $C_{n}[H]$, содержащих $v$, разобьем на три класса, класс $\alpha$-циклов, класс $\beta$-циклов и класс $\alpha \beta$-циклов. К первому классу отнесем те циклы, которые соответствуют простым циклам графа $P_{n}[H]$, содержащим вершину $\alpha\left(P_{n}[H]\right)$. Ко второму классу отнесем те циклы, которые соответствуют простым циклам графа $P_{n}[H]$, содержащим вершину $\beta\left(P_{n}[H]\right)$. К третьему классу отнесем те циклы, которые соответствуют простым путям графа $P_{n}[H]$, соединяющим вершину $\alpha\left(P_{n}[H]\right)$ и вершину $\beta\left(P_{n}[H]\right)$. Суммирование по $\alpha$-, $\beta$-и $\alpha \beta$-циклам обозначим соответственно $\sum_{\mathscr{C} \ni v}^{(\alpha)}, \sum_{\mathscr{C} \ni v}^{(\beta)}$ и $\sum_{\mathscr{C} \ni v}^{(\alpha \beta)}$.

Множество вершин графа $C_{n}[H]$, смежных с $v$, разобьем на два класса, класс $\alpha$-вершин и класс $\beta$-вершин. К первому классу отнесем вершины, соответствующие тем вершинам графа $P_{n}[H]$, которые смежны с вершиной $\alpha\left(P_{n}[H]\right)$. К второму классу отнесем вершины, соответствующие тем вершинам графа $P_{n}[H]$, которые смежны с вершиной $\beta\left(P_{n}[H]\right)$. Суммирование по $\alpha$-вершинам и по $\beta$-вершинам обозначим соответственно $\sum_{u \sim v}^{(\alpha)}$ и $\sum_{u \sim v}^{(\beta)}$.

Так как

$$
C_{n}[H]_{v}=P_{n}[H]_{\alpha \beta}, \quad \sum_{\mathscr{C} \ni v}^{(\alpha \beta)} C_{n}[H]_{\mathscr{C}}(\lambda)=s^{n},
$$

справедливы равенства

$$
\begin{aligned}
C_{n}[H](\lambda)= & \left(\lambda P_{n}[H]_{\alpha \beta}(\lambda)-\sum_{u \sim v}^{(\alpha)} C_{n}[H]_{u v}(\lambda)-2 \sum_{\mathscr{C} \ni v}^{(\alpha)} C_{n}[H]_{\mathscr{C}}(\lambda)\right) \\
& +\left(\lambda P_{n}[H]_{\alpha \beta}(\lambda)-\sum_{u \sim v}^{(\beta)} C_{n}[H]_{u v}(\lambda)-2 \sum_{\mathscr{C} \ni v}^{(\beta)} C_{n}[H]_{\mathscr{C}}(\lambda)\right) \\
& -\lambda P_{n}[H]_{\alpha \beta}(\lambda)-2 s^{n} \\
= & P_{n}[H]_{\beta}(\lambda)+P_{n}[H]_{\alpha}(\lambda)-\lambda P_{n}[H]_{\alpha \beta}(\lambda)-2 s^{n} .
\end{aligned}
$$

Совмещая это с формулами (11) и (12), получим следующее.

Для тех $\lambda$, для которых $s \neq 0$, справедливы равенства

$$
C_{n}[H](\lambda)=\left(P_{n-1}(t / s) t / s-2 P_{n-2}(t / s)-2\right) s^{n},
$$

а для тех $\lambda$, для которых $s=0$,

$$
C_{n}[H](\lambda)=t^{n} .
$$

К первому из этих равенств применим формулу из примера 1.

Теорема доказана.

\section{3. Следствия}

Сделаем несколько выводов о спектре (то есть о множестве собственных значений матрицы смежности) периодических графов. Спектр графа $G$ будем обозначать $\operatorname{Spec} G$. Ниже везде $H$ - двухкорневой граф с упорядоченными корнями.

Следствие 1. Пусть $\lambda \in \operatorname{Spec~} H$. Если $H_{\alpha}(\lambda) H_{\beta}(\lambda) \neq 0$, то

$$
P_{n}[H](\lambda)=-\lambda\left(H_{[\alpha \beta]}(\lambda)\right)^{n} P_{n-2}\left(H_{\alpha * \beta}(\lambda) / H_{[\alpha \beta]}(\lambda)\right),
$$

если $H_{\alpha}(\lambda) H_{\beta}(\lambda)=0$, то $P_{n}[H](\lambda)=0$. 
Следствие 2. Справедливо равенство

$$
C_{n}[H](\lambda)=\prod_{k=1}^{n}\left(H_{\alpha * \beta}(\lambda)-\left(2 \cos \frac{2 \pi k}{n}\right) H_{[\alpha \beta]}(\lambda)\right) .
$$

Если $\mu \in[-2,2]$, то все корни многочлена $H_{\alpha * \beta}(\lambda)-\mu H_{[\alpha \beta]}(\lambda)$ вещественные.

Доказательство. Первое утверждение вытекает из доказанной теоремы и равенства (см. $[1], \S 2.1)$

$$
C_{n}(\lambda)=\prod_{k=1}^{n}(\lambda-2 \cos (2 \pi k / n))
$$

Для доказательства второго утверждения заметим, что для каждого фиксированного $\mu \in 2 \cos (2 \pi(\mathbf{Q} \cap(0 ; 1]))$ все корни многочлена $H_{\alpha * \beta}(\lambda)-\mu H_{[\alpha \beta]}(\lambda)$ являются собственными значениями $C_{n}[H]$ для некоторого $n$, поэтому они вещественные. Множество же $2 \cos (2 \pi(\mathbf{Q} \cap(0 ; 1]))$ всюду плотно в $[-2 ; 2]$.

Множества $\bigcap_{n \geqslant 1} \operatorname{Spec} P_{n}[H]$ и $\bigcap_{n \geqslant 3} \operatorname{Spec} C_{n}[H]$ назовем, соответственно, цепным и циклическим спектрами графа $H$.

Следствие 3. Цепной спектр $H$ является пересечением Spec $H$ и множества всех корней многочлена $\lambda H_{\alpha}(\lambda) H_{\beta}(\lambda)$.

Циклический спектр $H$ совпадает $с$ множеством всех корней многочлена $H_{\alpha * \beta}(\lambda)-2 H_{[\alpha \beta]}(\lambda)$.

Доказательство. Цепной спектр $H$ есть подмножество Spec $H$. Пусть $\lambda_{0} \in \operatorname{Spec} H$. Из следствия 1 видно, что если $\lambda_{0}$ является корнем многочлена $H_{\alpha}(\lambda) H_{\beta}(\lambda)$, то оно принадлежит цепному спектру, а если не является, то принадлежит цепному спектру тогда и только тогда, когда

$$
\lambda_{0} P_{n-2}\left(H_{\alpha * \beta}\left(\lambda_{0}\right) / H_{[\alpha \beta]}\left(\lambda_{0}\right)\right)=0
$$

для любого натурального $n$. Последнее условие равносильно тому, что $\lambda_{0}=0$, так как $P_{0}(\lambda) \equiv 1$.

Множество $\mathscr{H}$ корней многочлена $H_{\alpha * \beta}(\lambda)-2 H_{[\alpha \beta]}(\lambda)$ включено в циклический спектр (это вытекает из следствия 2). Обратное включение следует из того, что

$$
\text { Spec } C_{3}[H] \cap \operatorname{Spec} C_{4}[H]=\mathscr{H}
$$

(это равенство также вытекает из следствия 2).

Отметим, что утверждение о цепном спектре доказано совместно с В. Ф. Субботиным.

Спектральным радиусом графа называется максимум модулей собственных значений этого графа.

Следствие 4. Пусть $R_{H}-$ максимум модулей корней многочлена

$$
\left(H_{\alpha * \beta}(\lambda)\right)^{2}-4\left(H_{[\alpha \beta]}(\lambda)\right)^{2} .
$$

Тогда спектральный радиус графа $C_{n}[H]$ не превосходит $R_{H}$. 
Доказательство. Предположим противное, пусть $\lambda^{*} \in \operatorname{Spec} C_{m}[H]$ и $\lambda^{*}>R_{H}$ (случай $\lambda^{*}<-R_{H}$ рассматривается аналогично). Из того, что $\lambda^{*}-$ не является корнем многочлена

$$
h(\lambda)=\left(H_{\alpha * \beta}(\lambda)\right)^{2}-4\left(H_{[\alpha \beta]}(\lambda)\right)^{2},
$$

но принадлежит спектру $C_{m}[H]$, следует, что $\lambda^{*}-$ корень многочлена $H_{\alpha * \beta}(\lambda)-(2 \cos (2 \pi k / m)) H_{[\alpha \beta]}(\lambda)$ для некоторого $k \in\{1, \ldots, m-1\} \backslash\{m / 2\}$. Если $H_{[\alpha \beta]}\left(\lambda^{*}\right)=0$, то $H_{\alpha * \beta}\left(\lambda^{*}\right)=0$, отсюда $h\left(\lambda^{*}\right)=0$. Получаем противоречие.

Пусть $H_{[\alpha \beta]}\left(\lambda^{*}\right) \neq 0$. Обозначим $f$ функцию, получающуюся из $H_{\alpha * \beta}(\lambda) / H_{[\alpha \beta]}(\lambda)$ устранением всех устранимых разрывов. Функция $f$ неограничена при $\lambda \rightarrow \infty$ и $\lim _{\lambda \rightarrow a} f(\lambda)=\infty$ для любой точки разрыва $a$. Так как $\left|f\left(\lambda^{*}\right)\right|<2$, найдется $\lambda^{* *}>\lambda^{*}$ такое, что $\left|f\left(\lambda^{* *}\right)\right|=2$, отсюда $h\left(\lambda^{* *}\right)=0$, поэтому $\lambda^{* *} \leqslant R_{H}$. Получаем противоречие.

\section{4. Открытые вопросы}

1. Исследовать вопрос об ограниченности спектрального радиуса графа $P_{n}[H]$ при росTe $n$.

2. Исследовать многочлены $H_{\alpha * \beta}(\lambda)$ и $H_{[\alpha \beta]}(\lambda)$, так как они играют ключевую роль в строении спектров периодических графов.

\section{Список литературы}

1. Цветкович Д., Дуб М., Захс Х., Спектры графов. Теория и применение. Наукова Думка, Киев, 1984.

2. Колмыков В. А., О суммировании по путям в спектральной теории графов. Дискретная математика (2001) 13, №4, 122-125.

Статья поступила 05.03.2002.

Переработанный вариант поступил 16.06.2003. 